\title{
GENETIC VARIABILITY AND HERITABILITY STUDIES IN RELATION TO SEED YIELD AND ITS COMPONENT TRAITS IN BLACKGRAM (VIGNA MUNGO(L.) HEPPER
}

\author{
CIRIVELA SHIRIN* \& G. M. LAL \\ Department of Genetics and Plant Breeding, Naini Agriculture Institute
}

Sam Higginbottom University of Agriculture, Technology and Sciences, Prayagraj,

Uttar Pradesh, India-211007

\begin{abstract}
During Kharif 2020, the current research was conducted at the Experimentation Centre, Department of Genetics and Plant Breeding, Naini Agricultural Institute, Sam Higginbottom University of Agriculture Technology and Science, Prayagraj, utilizing 38 genotypes. The genotypes were implanted in Randomized Block Design (RBD) with three replications. Analysis variability parameters, correlation coefficients, and path coefficients were applied to the average results. Based on the mean performance, high yield was found for the PKRU-03, KC-153, PDU-6, JU-2 showed higher than the check variety (T-9). Present experimental findings revealed that character's viz., number of clusters per plant, seed index, plant height, and number of primary branches per plant exhibited high GCV, PCV and heritability is coupled with high genetic advance as per cent of mean need to be given care while formulating breeding strategies for improvement of seed yield of blackgram.

KEYWORDS: Genetic variability, Heritability, Genetic advance
\end{abstract}

Received: Aug 11, 2021; Accepted: Aug 31, 2021; Published: Sep 09, 2021; Paper Id.: IJASRDEC202121

\section{INTRODUCTION}

Pulses are an essential source of protein for particularly vegetarian population of our country and they constitute a major part of our daily diet. Pulses have also been shown to improve soil fertility and crop productivity. The domestication and cultivation of staple food crops received more consideration than pulses. Pulses are perennially produced on low-fertility, moisture-stressed fields, thus genotypes are more adaptable to inadequate management, resulting in restricted output. This does not imply that pulses have little genetic potential; in fact, they may have better genetic potential than cereals. Blackgram [Vigna mungo (L.) Hepper], is a short-term grain legume that is economically valuable. It is a member of the family Fabaceae and subfamily Papillionaceae with chromosome no $2 n=22$. Its Centre of origin is India. Vigna radiate var. sublobata is thought to be the ancestor of blackgram.

\section{Area, Production, Productivity of Blackgram}

According to the 2019-20 census, blackgram occupies an area of 50.31 lakh/ha with production of 32.84 lakh tonnes and productivity of $653 \mathrm{~kg} / \mathrm{ha}$ whereas in Uttar Pradesh it occupies an area of $5.88 \mathrm{lakh} / \mathrm{ha}$ with production of 3.05 lakh tonnes and productivity of $520 \mathrm{~kg} / \mathrm{ha}$.

Source: directorate pulse development Ministry of agriculture \& farmers welfare 2019-20. 


\section{ECONOMIC IMPORTANCE}

Blackgram is an important pulse crop that is planted all over India. It's eaten as dal (whole or split, husked or unhusked) or perched. Urd is unique among pulses in that when soaked in water, it develops a mucilaginous pasty texture. It is consumed in a variety of ways across the North to South in preparation of different regular and popular dishes like vada, idli, dosa, halwa, imarti in combination with other food grains. Also used as nutritive fodder for mulch cattle.

India is the world's largest producer as well as consumer of Blackgram. It produces about 3.06 million tonnes of Blackgram annually from about 4.49 million hectares of area with an average productivity of $681.51 \mathrm{~kg}$ per hectare (Directorate of Economics and Statistics, Ministry of Agriculture and Farmers Welfare 2019-20).

The per capita availability of pulses has declined from $60.7 \mathrm{gm} /$ day in 1951 to $48 \mathrm{gm} / \mathrm{day}$ in 2019 as against the FAO/WHO"s recommendation of $80 \mathrm{gm} /$ day (Economic Survey, 2019-20). In this regard, the production potential of the blackgram crop can be increased by using targeted hybridization programmes to generate high yielding genotypes.

Seed yield being a complex character is influenced by several genetic factors interacting with the environment. A clear understanding of the association of plant characters with yield is necessary for a successful crop improvement programme. The correlation coefficient reflects the size and direction of the yield component relationship. Correlation coefficient analysis reveals the component characters on which selection can be based for yield improvement by measuring the mutual correlations between distinct plant character pairs. Character association aids in the development of productive genotypes by assisting in the formulation of an appropriate breeding plan.

\section{MATERIALS AND METHODS}

The experimental material for the present investigation consisted of 38 genotypes obtained from the Department of Genetics and Plant Breeding, SHUATS, Prayagraj. The experiment was carried out at the Field Experiment Centre of the Department of Genetics and Plant Breeding, SHUATS, Prayagraj during Kharif, 2020, which is nearly 5km away from Prayagraj city and is very near to river Yamuna. The site is located at $25.28 \mathrm{~N}$ latitude, $81.54 \mathrm{E}$ longitude and 98 meters above sea level. Soil texture Prayagraj comes under middle Gangetic plains (Agro climatic zone IV). The soil in this area is sandy loam, which is slightly alkaline. To raise a healthy crop, recommended cultural techniques were followed. Five competitive plants from each genotype were chosen at random for recording observations on thirteen features, including days to $50 \%$ flowering, days to $50 \%$ pod setting, days to maturity, plant height $(\mathrm{cm})$, number of primary branches per plant, number of clusters per plant, number of pods per plant, pod length $(\mathrm{cm})$, number of seeds per pod, biological yield per plant $(\mathrm{g})$, harvest index $(\%)$, seed index $(\mathrm{g})$ and seed yield per plant $(\mathrm{g})$. The conventional approach for analysis of variance was followed (Fisher, 1938). Genotypic coefficient of variation (GCV) and phenotypic coefficient of variation (PCV) (Burton, 1952), heritability (Burton and Devane, 1953), genetic advance (Johnson et al., 1955), were estimated.

\section{RESULTS AND DISCUSSION}

The analysis of variance for yield and yield contributing characters are presented in Table 1. The mean sum of squares due to genotypes was highly significant for all the traits studied in this investigation. Thus it reveals the presence of substantial amount variability among the genotypes under study.

The mean values, coefficient of variation (C.V), standard error of mean, critical differential (C.D) at 5\%, range of 38 genotypes and 13 quantitative characters are presented in Table 2, the perusal data revealed a wide range of variation 
for all traits studied viz., days to 50\% flowering range from 41.00 days (KC-153) to 49.66 days (VBN-08), days to 50\% pod setting range from 52.667 days (PLU-856) to 62.733 days (PDU-103), pod setting range from 62.033 days (IC-91-567) to 71.8 days (ADT-3), plant height range from PLU-1016 $(45.60 \mathrm{~cm})$ to DH- $85-2(83.193 \mathrm{~cm})$, number of primary branches range from BG-369 (2.733) to PLU-302 (9.800), number of cluster per plant range from BG-369(3.667) to P-1 (27.867), number of pods per plant range from IC-91-567 (9.067) to PKRU-03 (47.967), number of seeds per pod range from PKGU-03 (4.833) to TBG-104 (7.867), pod length range from PDU-6 (3.6) to BG-369 (4.76), seed index ranged from PKGU-03 (2.367) to TBG-104 (5.800), biological yield ranged from LBG-752(7.013) to PKRU-03(21.743), harvest index ranged from LBG-648(21.133) to BG-369(46.000), seed yield per plant ranged from PLU-302 (3.013) to IPU-9916(4.533).

\section{Genotypic and Phenotypic Coefficient of Variation}

The estimates of variability parameters for various yield and yield related traits are presented in Table 3 . An estimate of GCV and PCV revealed the phenotypic coefficient of variation was higher than genotypic coefficient of variation, which indicates the presence of environment effects on expression on character studied. Higher magnitudes of PCV were recorded for number of cluster per plant (47.541) followed by number of branches per plant (42.984), number of pods per plant (40.366), harvest index (27.767). Moderate magnitude of PCV were recorded for seed yield per plant (26.73) followed by plant height (24.003), biological yield (21.454), seed index (20.233) no. of seeds per pod (11.975). Low magnitude of PCV were recorded for plant length (7.239) followed by days to $50 \%$ pod setting (5.465), days to $50 \%$ flowering (4.479), days to maturity (2.564).

Higher magnitude of GCV were recorded for number of cluster per plant (40.298) followed by number of branches per plant (39.994), number of pods per plant (34.997), plant height (20.025), harvest index (19.852). Moderate magnitudes of GCV were recorded for seed yield per plant (19.784), seed index (17.816), biological yield per plant (12.59), number of seeds per pod (9.108). Low magnitudes of GCV were recorded for days to 50\% pod setting (4.693), pod length (4.35), days to $50 \%$ flowering (3.36), days to maturity (2.225).

\section{Heritability (\%) and Expected Genetic Advance}

The perusal of table 3 revealed the estimates of heritability (\%) in broad sense for 13 characters studied, which ranged from $(34.4 \%)$ to $(86.6 \%)$. Number of branches per plant $(86.6 \%)$, seed index $(77.5 \%)$, showed high heritability. Moderate heritability days to maturity $(75.3 \%)$ when compared to other genetic parameters for heritability. Low heritability was recorded for number of pods per plant $(75.1 \%)$, days to $50 \%$ pod setting $(73.7 \%)$, number of cluster per plant $(71.8 \%)$, plant height $(69.6 \%)$, number of seeds per pod (57.8\%), days to $50 \%$ flowering $(56.3 \%)$, seed yield per plant $(54.8 \%)$, harvest index $(51.1 \%)$, pod length $(36.1 \%)$, biological yield per plant $(34.4 \%)$.

High estimate of genetic advance as per cent of mean was recorded for number of branches per plant (76.658), number of cluster per plant (70.364), number of pod per plant (62.433), plant height (34.414). Moderate estimate of genetic advance as percent of mean was recorded for seed index (32.315), seed yield per plant (30.164), harvest index (29.239), biological yield per plant (15.221), number of seeds per pod (14.271). Low estimate of genetic advance as percent of mean was recorded for days to $50 \%$ pod setting (8.3), pod length (5.386), days to 50\% flowering (5.192), days to maturity (3.978). This indicated that in blackgram, there is a higher chance to increase yield and characteristics.

\section{CONCLUSIONS}


The experimental results conducted that based on field performance of 38 genotypes of blackgram the highest yield was recorded in PKRU-03(7.627g) and identified as desirable genotype with seed yield per plant compared with T-9(4.580g). All genotypes mature early than check variety. The higher magnitude of GCV and PCV were recorded for number of clusters per plant and number of primary branches per plant for heritability and genetic advance were plant height. Thus, priority should be given to these characters for yield improvement in blackgram.

Table 1: Analysis of Variance for 13 different Quantitative Characters in 38 Genotypes of blackgram

\begin{tabular}{|l|c|c|c|}
\hline \multirow{2}{*}{ CHARACTERS } & \multicolumn{3}{|c|}{ MEAN SUM OF SQUARES } \\
\cline { 2 - 4 } & Replication & Treatments & Error \\
\cline { 2 - 4 } & $(\mathrm{df}=2)$ & $(\mathrm{df}=37)$ & $(\mathrm{df}=74)$ \\
\hline Days to 50\% flowering & 25.15 & 8.75 & 1.80 \\
\hline Days to 50\% pod setting & 16.40 & 24.29 & 2.57 \\
\hline Days to maturity & 17.31 & 7.97 & 0.78 \\
\hline Plant height & 41.11 & 471.55 & 59.93 \\
\hline $\begin{array}{l}\text { No. of primary branches per } \\
\text { plant }\end{array}$ & 4.87 & 13.53 & 0.66 \\
\hline No. of clusters per plant & 44.81 & 83.38 & 9.63 \\
\hline No. of pods per plant & 43.17 & 241.39 & 24.04 \\
\hline No. of seeds per plant & 2.28 & 1.24 & 0.24 \\
\hline Pod length & 0.18 & 0.17 & 0.06 \\
\hline Seed index & 0.051 & 1.93 & 0.17 \\
\hline Biological yield & 9.27 & 24.70 & 5.97 \\
\hline Harvest Index & 79.48 & 96.07 & 37.29 \\
\hline Seed yield per plant & 3.27 & 2.78 & 0.60 \\
\hline
\end{tabular}

$* * 1 \%$ Level of significance and * $5 \%$ Level of significance

Table 2: Mean Performances of 38 blackgram Genotypes for 13 Quantitative Characters

\begin{tabular}{|c|c|c|c|c|c|c|c|c|c|c|c|c|c|c|}
\hline $\begin{array}{l}\text { Sl. } \\
\text { No. }\end{array}$ & Geitotypes & $\begin{array}{c}\text { Days to } \\
\mathbf{5 0 \%} \\
\text { Flowering }\end{array}$ & $\begin{array}{l}\text { Days to } \mathbf{5 0 \%} \\
\text { Pod Setting }\end{array}$ & $\begin{array}{l}\text { Days to } \\
\text { Maturity }\end{array}$ & $\begin{array}{c}\text { Plant } \\
\text { height } \\
(\mathrm{cm})\end{array}$ & $\begin{array}{c}\text { Number of } \\
\text { Primary } \\
\text { Branches } \\
\text { Per Plant }\end{array}$ & $\begin{array}{c}\text { Number of } \\
\text { Clusters Per } \\
\text { Plant }\end{array}$ & $\begin{array}{l}\text { Number of } \\
\text { Pods Per } \\
\text { Plant }\end{array}$ & $\begin{array}{l}\text { Number of } \\
\text { Seeds Per } \\
\text { Pod }\end{array}$ & $\begin{array}{c}\text { Pod } \\
\text { Length } \\
(\mathrm{cm})\end{array}$ & $\begin{array}{l}100 \text { seed } \\
\text { Weight } \\
(\mathrm{g})\end{array}$ & $\begin{array}{c}\text { Harrest } \\
\text { Index } \\
(\%)\end{array}$ & $\begin{array}{l}\text { Biological } \\
\text { Yield Per } \\
\text { Plant }(g)\end{array}$ & $\begin{array}{l}\text { Seed } \\
\text { Yield Per } \\
\text { Plant }(g)\end{array}$ \\
\hline 1 & PKRU-03 & 45.667 & 55.000 & 69.260 & 81.100 & 3.800 & 14.867 & 47.967 & 5.600 & 4.613 & 3.400 & 35.363 & 21.743 & 7.627 \\
\hline 2 & LBG -648 & 44.333 & 56.667 & 69.390 & 79.953 & 3.167 & 5.267 & 18.667 & 5.600 & 4.507 & 3.700 & 21.133 & 16.777 & 3.520 \\
\hline 3 & $\mathrm{FDU}-6$ & 46.333 & 56.333 & 69.370 & 79.920 & 3.367 & 15.300 & 44.200 & 5.667 & 3.647 & 3.300 & 37.060 & 15.787 & 5.867 \\
\hline 4 & D $-85-2$ & 46.000 & 55.000 & 69.017 & 83.193 & 3.433 & $4.7 p 0$ & 23.100 & 5.833 & 4.420 & 3.633 & 25.533 & 13.283 & 3.403 \\
\hline 5 & $\mathrm{KC}-153$ & 41.000 & 53.667 & 68.580 & 75.460 & 3.200 & 16.067 & 43.267 & 5.900 & 4.493 & 4.500 & 34.107 & 17.703 & 6.053 \\
\hline 6 & PGRU-99022 & 45.667 & 56.000 & 69.583 & 73.967 & 3.300 & 6.567 & 25.400 & 5.500 & 4.360 & 3.167 & 33.290 & 12.730 & 4.240 \\
\hline 7 & VBN-08 & 49.667 & 57.000 & 70.213 & 68.693 & 2.767 & 6.567 & 28.333 & 5.367 & 4.483 & 4.500 & 31.780 & 15.570 & 4.853 \\
\hline 8 & S.TP-02 & 44.667 & 54.000 & 69.467 & 75.187 & 3.567 & 7.133 & 26.467 & 5.333 & 4.493 & 4.333 & 29.580 & 13.050 & 3.833 \\
\hline 9 & BG.369 & 42.000 & 53.333 & 68.413 & 70.407 & 2.733 & 3.667 & 18.667 & 6.100 & 4.760 & 4.367 & 46.000 & 9.720 & 4.247 \\
\hline 10 & PLU-557 & 46.000 & 55.333 & 70.070 & 56.607 & 3.033 & 9.667 & 27.433 & 6.067 & 4.447 & 4.700 & 36.383 & 9.943 & 3.587 \\
\hline 11 & PKGU -03 & 43.667 & 55.000 & 70.133 & 46.200 & 2.967 & 7.667 & 21.833 & 4.833 & 4.217 & 2.367 & 35.097 & 11.043 & 3.727 \\
\hline 12 & $\mathrm{PLU}-856$ & 47.000 & 52.667 & 68.310 & 76.900 & 2.933 & 7.400 & 23.267 & 6.600 & 4.293 & 3.467 & 45.657 & 12.173 & 5.223 \\
\hline 13 & PLU - 1050 & 45.800 & 56.933 & 69.163 & 52.800 & 3.533 & 16.000 & 25.600 & 6.400 & 4.460 & 4.100 & 30.130 & 12.417 & 3.740 \\
\hline 14 & IAU-1 & 45.867 & 57.333 & 69.473 & 51.467 & 3.933 & 6.400 & 11.267 & 5.867 & 4.080 & 4.067 & 31.733 & 10.460 & 3.320 \\
\hline 15 & IPE-99-16 & 45.733 & 57.133 & 70.390 & 55.533 & 5.533 & 20.467 & 33.600 & 6.133 & 4.313 & 6.200 & 36.407 & 12.487 & 4.533 \\
\hline 16 & PLU -1016 & 43.600 & 53.333 & 69.163 & 45.600 & 3.600 & 11.067 & 16.400 & 7.067 & 4.587 & 4.133 & 40.370 & 11.607 & 4.607 \\
\hline 17 & XP-16 & 46.533 & 58.200 & 70.213 & 52.467 & 3.733 & 15.333 & 26.733 & 6.400 & 4.607 & 5.367 & 36.563 & 13.210 & 4.833 \\
\hline 18 & PDV -2 & 45.867 & 57.333 & 69.370 & 51.220 & 7.333 & 15.133 & 22.843 & 6.400 & 4.200 & 4.700 & 31.377 & 10.613 & 3.327 \\
\hline 19 & LBG - 752 & 44.533 & 55.600 & 69.393 & 47.667 & 5.133 & 11.267 & 15.867 & 6.533 & 4.687 & 5.733 & 46.597 & 7.013 & 3.260 \\
\hline 20 & IC $-91-567$ & 47.333 & 59.133 & 62.033 & 58.600 & 9.033 & 8.467 & 9.067 & 6.133 & 4.333 & 4.567 & 31.047 & 9.940 & 3.087 \\
\hline 21 & $\mathrm{U}-2$ & 44.933 & 56.000 & 69.017 & 48.667 & 5.733 & 14.567 & 23.767 & 6.400 & 4.433 & 4.000 & 34.863 & 15.083 & 5.233 \\
\hline 22 & BGP -21-28 & 43.467 & 54.333 & 68.413 & 48.867 & 5.600 & 10.933 & 18.400 & 6.533 & 4.400 & 3.033 & 36.757 & 13.510 & 4.967 \\
\hline
\end{tabular}




\begin{tabular}{|c|c|c|c|c|c|c|c|c|c|c|c|c|c|c|}
\hline $\begin{array}{l}\text { Sl. } \\
\text { No. }\end{array}$ & Genotypes & $\begin{array}{c}\text { Days to } 50 \% \\
\text { Flowering }\end{array}$ & $\begin{array}{l}\text { Days to 50\% } \\
\text { Pod Setting }\end{array}$ & $\begin{array}{l}\text { Days to } \\
\text { Maturity }\end{array}$ & $\begin{array}{l}\text { Plant } \\
\text { Height } \\
\text { (cm) }\end{array}$ & $\begin{array}{l}\text { Primary } \\
\text { Branches } \\
\text { Per Plant }\end{array}$ & $\begin{array}{l}\text { Clusters } \\
\text { Per Plant }\end{array}$ & $\begin{array}{c}\text { Number of } \\
\text { Pods Per } \\
\text { Plant }\end{array}$ & $\begin{array}{l}\text { Number } \\
\text { of Seeds } \\
\text { Per Pod }\end{array}$ & $\begin{array}{l}\text { Pod length } \\
(\mathrm{cm})\end{array}$ & $\begin{array}{l}100 \text { seed } \\
\text { Weight } \\
\text { (g) }\end{array}$ & $\begin{array}{l}\text { Harrest } \\
\text { Index } \\
(\%)\end{array}$ & $\begin{array}{l}\text { Biological } \\
\text { yield }(g)\end{array}$ & $\begin{array}{l}\text { Seed } \\
\text { Yield Per } \\
\text { Plant }(\mathrm{g})\end{array}$ \\
\hline 23 & LBG - 20 & 47.067 & 59.067 & 69.393 & 49.667 & 7.667 & 14.800 & 24.533 & 6.733 & 4.720 & 4.867 & 40.507 & 13.277 & 5.220 \\
\hline 24 & PLU-144 & 45.867 & 57.267 & 70.070 & 34.067 & 4.133 & 8.867 & 19.133 & 6.733 & 4.287 & 4.133 & 41.023 & 12.163 & 4.987 \\
\hline 25 & VBN - 6 & 45.400 & 56.800 & 70.213 & 43.933 & 5.667 & 9.533 & 15.800 & 6.533 & 4.373 & 3.900 & 41.977 & 11.063 & 4.613 \\
\hline 26 & ADT-3 & 47.067 & 61.467 & 71.800 & 46.533 & 6.267 & 15.867 & 29.133 & 6.933 & 4.693 & 4.000 & 35.220 & 11.893 & 4.200 \\
\hline 27 & DH $-85-5$ & 45.267 & 62.667 & 71.370 & 57.267 & 7.400 & 14.467 & 20.933 & 6.400 & 4.480 & 4.233 & 39.173 & 7.127 & 2.740 \\
\hline 28 & LBG -645 & 47.067 & 61.467 & 70.213 & 47.267 & 9.067 & 20.800 & 33.133 & 6.400 & 4.287 & 5.033 & 37.133 & 10.130 & 3.753 \\
\hline 29 & PLU - 302 & 45.200 & 59.133 & 69.863 & 62.200 & 9.800 & 14.133 & 18.267 & 6.267 & 4.040 & 4.167 & 30.927 & 9.750 & 3.013 \\
\hline 30 & MASH - 338 & 47.067 & 62.133 & 71.487 & 50.467 & 8.667 & 22.667 & 32.400 & 7.667 & 4.927 & 4.133 & 39.353 & 11.177 & 4.400 \\
\hline 31 & P-1 & 47.200 & 61.800 & 71.487 & 55.467 & 9.400 & 27.867 & 41.867 & 6.067 & 4.133 & 4.700 & 38.467 & 9.790 & 3.767 \\
\hline 32 & SPS - 40 & 44.133 & 56.800 & 69.360 & 50.200 & 7.800 & 12.133 & 19.600 & 6.400 & 4.133 & 4.533 & 30.253 & 12.603 & 3.780 \\
\hline 33 & TBG-104 & 45.600 & 62.533 & 70.213 & 58.067 & 5.533 & 12.933 & 19.867 & 7.867 & 4.873 & 5.800 & 25.987 & 15.460 & 3.753 \\
\hline 34 & SN-2115 & 42.267 & 58.000 & 71.800 & 48.783 & 4.867 & 13.133 & 22.200 & 7.267 & 4.580 & 4.167 & 29.923 & 14.103 & 4.273 \\
\hline 35 & PDU - 103 & 45.067 & 62.733 & 71.370 & 51.200 & 4.733 & 11.400 & 20.267 & 7.067 & 4.360 & 5.500 & 36.290 & 13.277 & 4.727 \\
\hline 36 & $\mathrm{C} 0-6$ & 44.733 & 59.533 & 70.510 & 55.567 & 5.667 & 12.600 & 17.400 & 6.467 & 4.380 & 3.667 & 30.667 & 16.500 & 4.980 \\
\hline 37 & IPU - 1070 & 42.267 & 55.000 & 69.737 & 65.517 & 4.667 & 6.400 & 11.867 & 7.200 & 4.547 & 5.267 & 31.223 & 12.527 & 3.907 \\
\hline \multirow[t]{10}{*}{38} & T-90 & 45.400 & 56.800 & 66.967 & 66.133 & 4.000 & 15.467 & 26.200 & 6.867 & 4.453 & 4.200 & 41.050 & 11.590 & 4.580 \\
\hline & Mean & 45.325 & 57.330 & 69.587 & 58.495 & 5.178 & 12.304 & 24.335 & 6.346 & 4.424 & 4.306 & 35.158 & 12.587 & 4.310 \\
\hline & C.V. & 2.962 & 2.802 & 1.274 & 13.235 & 15.751 & 25.225 & 20.150 & 7.775 & 5.786 & 9.591 & 17.371 & 19.414 & 17.975 \\
\hline & F ratio & 4.861 & 9.416 & 10.149 & 7.867 & 20.342 & 8.657 & 10.039 & 5.117 & 2.696 & 11.351 & 2.576 & 4.137 & 4.634 \\
\hline & F Prob. & 0.000 & 0.000 & 0.000 & 0.000 & 0.000 & 0.000 & 0.000 & 0.000 & 0.000 & 0.000 & 0.000 & 0.000 & 0.000 \\
\hline & S.E. & 0.775 & 0.927 & 0.512 & 4.470 & 0.471 & 1.792 & 2.831 & 0.285 & 0.148 & 0.239 & 3.526 & 1.411 & 0.447 \\
\hline & C.D. $5 \%$ & 2.184 & 2.613 & 1.443 & 12.596 & 1.327 & 5.049 & 7.978 & 0.803 & 0.416 & 0.672 & 9.936 & 3.975 & 1.260 \\
\hline & C.D. $1 \%$ & 2.898 & 3.467 & 1.914 & 16.713 & 1.761 & 6.700 & 10.586 & 1.065 & 0.553 & 0.892 & 13.184 & 5.275 & 1.672 \\
\hline & Range Lowrest & 41.000 & 52.667 & 62.033 & 34.067 & 2.733 & 3.667 & 9.067 & 4.833 & 3.647 & 2.367 & 21.133 & 7.013 & 2.740 \\
\hline & Range Highest & 49.667 & 62.733 & 71.800 & 83.193 & 9.800 & 27.867 & 47.967 & 7.867 & 4.927 & 6.200 & 46.597 & 21.743 & 7.627 \\
\hline
\end{tabular}

Table 3: Genetic Parameters for 13 Characters of 38 blackgram Genotypes

\begin{tabular}{|c|c|c|c|c|c|c|c|}
\hline Charactero & $\begin{array}{c}\text { Genotypic } \\
\text { Variance }(\mathrm{Vg}) \propto\end{array}$ & $\begin{array}{c}\text { Phenotypic } \\
\text { Variance }(\text { Vp) } \alpha\end{array}$ & GCVo & PCVa & $\begin{array}{c}\text { Heritability } \\
\text { (\%)Broad'*-sensea }\end{array}$ & GAa & GA as $\%$ 'meano \\
\hline Days to $50 \%$ floweringa & $2.319 \alpha$ & 4.1210 & $3.36 \mathrm{~d}$ & 4.4790 & $56.3 \alpha$ & 2.3530 & 5.1920 \\
\hline Days to $50 \%$ pod settingo & $7.238 \alpha$ & $9.817 \alpha$ & 4.6930 & 5.4650 & $73.7 \alpha$ & 4.7580 & 8.30 \\
\hline Days to maturityo & 2.3980 & $3.184 \alpha$ & 2.2250 & $2.564 a$ & $75.3 \alpha$ & $2.768 \mathrm{~d}$ & $3.978 \propto$ \\
\hline Plant heighto & 137.2060 & $197.145 \alpha$ & $20.025 \alpha$ & $24.003 \alpha$ & 69.60 & $20.13 \alpha$ & $34.414 \alpha$ \\
\hline No. of branches per planto & $4.289 a$ & 4.9540 & $39.994 \alpha$ & $42.984 \alpha$ & 86.60 & 3.9690 & $76.658 \alpha$ \\
\hline No. of cluster perplanto & $24.585 \alpha$ & $34.219 \alpha$ & $40.298 \alpha$ & 47.54lø & 71.80 & 8.6580 & $70.364 \alpha$ \\
\hline No. of pods per planto & 72.4490 & $96.494 a$ & 34.9970 & $40.366 \mathrm{a}$ & 75.10 & $15.193 \mathrm{a}$ & 62.4330 \\
\hline No. of seeds per poda & 0.3340 & $0.577 \alpha$ & 9.1080 & $11.975 \propto$ & $57.8 \alpha$ & 0.9060 & 14.2710 \\
\hline Pod lengtho & $0.037 x$ & $0.103 \alpha$ & 4.350 & 7.2390 & 36.10 & $0.238 \alpha$ & 5.3860 \\
\hline Seed index 0 & 0.5890 & 0.7590 & 17.8160 & $20.233 a$ & $77.5 \alpha$ & 1.3920 & $32.315 \alpha$ \\
\hline Biologicalyielda & 19.5940 & $56.891 \alpha$ & 12.590 & $21.454 \alpha$ & $34.4 \alpha$ & 5.3510 & 15.2210 \\
\hline Harvest index $\propto$ & $6.244 a$ & $12.215 \alpha$ & $19.852 \alpha$ & $27.767 x$ & 51.10 & $3.68 \alpha$ & 29.23900 \\
\hline Seed yield per planto & $0.727 x$ & $1.327 \alpha$ & $19.784 \alpha$ & $26.73 \alpha$ & 54.80 & 1.30 & $30.164 \alpha$ \\
\hline
\end{tabular}

\section{REFERENCES}

1. Fisher, R. A. (1936). Statistical tables for biological, agricultural and mendelian inheritance. France Royal Society of Edinburgh, 52:399-433.

2. Isaacs, S, Jebaraj, M. S. and Ganesh, S. K. (2000). Estimation of heritability, genetic variability and path analysis in blackgram (Vigna mungo (L.) Hepper), Research on crops, 1(3):314-316.

3. Konda, C. R., Salimath, P. M., and Mishra, M. N. (2009). Genetic variability studies for productivity and its components in blackgram (Vigna mungo (L.) Hepper). Legume Research, 32 (1): 59-61. 
4. Kumar, B. and Mishra, M. N. (2004). Genetic variability in blackgram germplasm. Annals of Agricultural Research, 25: 406407.

5. Lad, D. B., Pande, P. B. and Jagtap, P. K. (2010). Genetic variability studies in blackgram (Vigna mungo L. Hepper). Journal of Maharashtra Agricultural Universities, 35 (2): 325-327.

6. Meshram, M. P., Sonone, N. G. and Patil, A. N. (2012). Genetic variability studies in urdbean. PKV Research Journal, 36 (2):22-26.

7. Natarajan, C, and Rathinasamy, R. (1999). Genetic variability, correlation blackgram (Vigna mungo (L.) Hepper), Madras Agricultural Journal, 86: 4-6.

8. Pervin, M. A., Polash, M. F. M. B., Rahman, S. M and Deb, A. C. (2007). Study of genetic variability and $G X$ E interaction of some quantitative traits in blackgram (Vigna mungo L. Hepper). Journal of Biological Sciences, 7(1) 160-175.

9. Ramprasad, P. V. S., Reddy, P. N., Reddy, K. R., Reddy, P. R. Reddy, G. L. K. and Reddy, M. V. (1989). Heritability and genetic advance in certain crosses of Blackgram (Vigna mungo (L.) Hepper). Journal of Research Andhra Pradesh Agricultural University. 17:60-61.

10. Sarkar, G., Panda, S. and Senapati, B.K. (2006). Genetic variability and character association in blackgram (Vigna mungo L. Hepper). Journal of Arid Legumes, 3(1): 65-70.

11. Toppo, N.A., Nair, S.K., Nanda, H.C. and Sopan (2019). Genetic variability studies on yield and yield contributing traits in blackgram (Vigna mungo (L.) Hepper).Journal of Pharmacognosy and Phytochemistry, 8(6): 1713-1716.

12. Vinoth, $\boldsymbol{R}$. and Jayamani P. (2014). Genetic variability studies for yield and its component traits in inter sub specific RIL population of blackgram (Vigna mungo (L.) Hepper). Trends in Biosciences. 7: 243-245.

13. Wani, S.K., Sridevi, O. and Salimath, P.M. (2007). Studies on genetic variability, correlation and path analysis in chilli (Capsicum annum). Annals of Biological Sciences, 23(2): 117-121.

14. Wilkis, S.S. (1932). Certain generalizations in the analysis of variance. Biometrics, 24: 471. 